\title{
Impact of Circulating and Tissue Biomarkers in Adjuvant and Neoadjuvant Therapy for High-Risk Melanoma: Ready for Prime Time?
}

\author{
Alice Indini ${ }^{1} \cdot$ Fausto Roila ${ }^{2} \cdot$ Francesco Grossi $^{3} \cdot$ Daniela Massi $^{4} \cdot$ Mario Mandalà ${ }^{2}$
}

Accepted: 4 May 2021 / Published online: 25 May 2021

(c) The Author(s) 2021

\begin{abstract}
The prognosis of patients with metastatic melanoma has substantially improved over the last years with the advent of novel treatment strategies, mainly immune checkpoint inhibitors and BRAF and MEK inhibitors. Given the survival benefit provided in the metastatic setting and the evidence from prospective clinical trials in the early stages, these drugs have been introduced as adjuvant therapies for high-risk resected stage III disease. Several studies have also investigated immune checkpoint inhibitors, as well as BRAF and MEK inhibitors, for neoadjuvant treatment of high-risk stage III melanoma, with preliminary evidence suggesting this could be a very promising approach in this setting. However, even with new strategies, the risk of disease recurrence varies widely among stage III patients, and no available biomarkers for predicting disease recurrence have been established to date. Improved risk stratification is particularly relevant in this setting to avoid unnecessary treatment for patients who have minimum risk of disease recurrence and to reduce toxicities and costs. Research for predictive and prognostic biomarkers in this setting is ongoing to potentially shed light on the complex interplay between the tumor and the host immune system, and to further personalize treatment. This review provides an insight into available data on circulating and tissue biomarkers, including the tumor microenvironment and associated gene signatures, and their predictive and prognostic role during neoadjuvant and adjuvant treatment for cutaneous high-risk melanoma patients.
\end{abstract}

Mario Mandalà

mario.mandala@unipg.it

\section{Key Points}

Stage III melanoma patients are a heterogeneous population and biomarkers for disease recurrence have not been established to date.

Biomarkers for a strong adaptive immune response seem to identify patients who derive clinical benefit from adjuvant therapy.

Results in a neoadjuvant setting need to be implemented and prospectively confirmed to be employed in everyday clinical practice.

1 Medical Oncology Unit, Department of Internal Medicine, Fondazione IRCCS Ca' Granda Ospedale Maggiore Policlinico, Milan, Italy

2 Unit of Medical Oncology, Department of Surgery and Medicine, University of Perugia, Perugia, Italy

3 Unit of Medical Oncology, Ospedale di Circolo e Fondazione Macchi, Università dell'Insubria, Varese, Italy

4 Section of Pathological Anatomy, Department of Health Sciences, University of Florence, Florence, Italy 


\section{Introduction}

Cutaneous melanoma is an aggressive disease and the rates of recurrence are proportional to disease stage at diagnosis [1]. Early-stage disease [i.e. stage I and IIA, according to the recently updated classification of the American Joint Committee on Cancer (AJCC) 8th edition] is managed with radical surgery and usually does not require further treatment [2]. Patients with sentinel lymph node involvement (i.e. stage III) are at higher risk for recurrence after surgical resection, and many of them will ultimately die from metastatic melanoma [3]. For this reason, patients with stage III melanoma benefit from adjuvant systemic therapy, aiming to reduce the risk of disease relapse and potentially improve survival rates. In selected patients with high-risk stage III melanoma, neoadjuvant treatment might lead to increased disease control and better outcomes after surgery, however this approach is not considered as standard of care. Overall, stage III melanoma patients are a heterogeneous population, with 5-year survival rates ranging from 80 to $85 \%$ in stage IIIA disease, to $32 \%$ for stage IIID disease [2]. The risk of disease recurrence varies widely in stage III patients and no available biomarkers for predicting disease recurrence have been established to date.

This review summarizes the most recent results from studies exploring predictive and prognostic biomarkers in the neoadjuvant and adjuvant settings, providing a focus on ongoing research in this field that in turn will help selection and treatment decision making in high-risk melanoma patients.

The referenced papers were selected through a PubMed search performed on 5 April 2021 using the following search terms: melanoma and biomarkers, and adjuvant, or neoadjuvant, and immunotherapy, or anti-programmed cell death 1 (PD1), or anti-cytotoxic T-lymphocyte antigen 4 (CTLA-4), and targeted therapy, or BRAF. The ClinicalTrials.gov database was searched to identify ongoing clinical trials in neoadjuvant and adjuvant settings in cutaneous melanoma, with exploratory analysis evaluating circulating and tissue biomarkers.

\section{Adjuvant Therapy in Melanoma}

The prognosis of patients with advanced unresectable stage III/metastatic stage IV melanoma has significantly improved over the last years with the advent of novel systemic therapies, namely immunotherapy and targeted therapy [4-9]. The first strategy includes unleashing the immune response through immune checkpoint inhibitors, with antibodies targeting the cytotoxic T-lymphocyte antigen 4 (CTLA-4) and programmed cell death 1 (PD1) [4-6]. The second strategy consists of targeting the mitogen-activated protein kinase (MAPK) pathway (i.e. the combination of BRAF and MEK inhibitors), which is constitutively activated in approximately $50 \%$ of cutaneous melanoma patients, namely those harboring a BRAFV600 mutation [7-9]. Given the survival benefit provided by these drugs in the metastatic setting, in recent years efforts have been made to evaluate their role as adjuvant treatment for high-risk resected disease [10]. The first immunotherapy to demonstrate a significant improvement in relapsefree survival (RFS) and overall survival (OS) compared with placebo was the anti-CTLA4 antibody ipilimumab in the European Organisation for Research and Treatment of Cancer (EORTC) 18071/CA184-029 trial [11, 12]. However, ipilimumab was associated with a high rate of serious adverse events (SAEs), leading to early treatment discontinuation in a substantial proportion of patients and to treatment-related deaths (five patients, 1.1\%) [11]. Indeed, anti-PD1 antibodies were demonstrated to be more effective and less toxic than ipilimumab in metastatic disease [5, 6]. The CheckMate 238 trial compared nivolumab with high-dose ipilimumab in patients with high-risk, radically resected stage IIIB/IIIC or stage IV melanoma [13]. After a minimum of 4 years' follow-up, adjuvant nivolumab demonstrated a significant improvement in RFS and distant metastases-free survival (DMFS) compared with ipilimumab [14]. Nivolumab exhibited a favorable toxicity profile, with fewer patients experiencing treatment-related SAEs or discontinuing therapy due to any treatment-related toxicity. Furthermore, there were no treatment-related deaths in the cohort of patients treated with nivolumab [13, 14]. Similarly, adjuvant pembrolizumab for resected high-risk stage III melanoma resulted in significantly longer RFS than placebo in the EORTC 1325-MG/KEYNOTE-054 trial [15], with no reports of new toxic effects at 3 years' follow-up [16]. In both trials, the survival improvement of adjuvant anti-PD1 was consistent across all patient subgroups, regardless of disease stage and $B R A F$ mutational status [13, 15].

Adjuvant treatment options for patients with $B R A F$ mutant melanoma also include the combination of a BRAF inhibitor (dabrafenib) with an MEK inhibitor (trametinib). In the COMBI-AD trial, adjuvant dabrafenib and trametinib significantly improved RFS versus placebo in patients with high-risk stage III cutaneous melanoma with $B R A F$ V600E or V600K mutations $[17,18]$. In this trial, toxicity was consistent with previous data regarding targeted therapy in the metastatic setting, and no new toxic effect was observed. To date, there is no clinical trial evidence directly comparing adjuvant immunotherapy and targeted therapy. Indirect comparisons of the available trials suggest that the incidence 
of primary resistance (i.e. disease relapse or death within the first 6 months of adjuvant treatment) is more frequently observed during anti-PD1 therapy than with targeted therapy. However, 4-year RFS seems to be similar between the two treatment strategies, suggesting there may be multiple factors determining response or resistance mechanisms to adjuvant treatment [19].

Improved risk stratification is particularly relevant in the setting of adjuvant treatment, for both toxicities and costs related to novel treatments. Therefore, patients' selection represents the mainstay of adjuvant treatment in melanoma, to avoid unnecessary treatment for patients who have minimum risk of disease recurrence. There are no specific clinical or biological characteristics that identify which patients will benefit more from available adjuvant treatments, but several biomarkers have been studied with promising preliminary results.

\section{Neoadjuvant Therapy in Melanoma}

There is a strong rationale supporting the role of neoadjuvant immunotherapy in surgically resectable melanoma. Preclinical evidence demonstrated that immune checkpoint inhibitors administered before surgery correlated with better survival compared with adjuvant treatment [20]. Pathologic complete response ( $\mathrm{pCR}$ ) rate to neoadjuvant therapy has a prognostic role and can also be used as a tool to determine adjuvant treatment and follow-up schedules [21]. Moreover, the comparison of tumor tissues obtained before and after surgery can reveal important information regarding potential biomarkers for response and resistance to treatment [20]. It is also important to underline that approximately $40 \%$ of patients with stage III melanoma will relapse within the first 3 years despite being treated with adjuvant therapy (either with targeted therapy or immunotherapy), and up to $25 \%$ of patients experience early relapse before even starting adjuvant treatment [22].

To date, several trials have been conducted to test neoadjuvant immune checkpoint inhibitors in high-risk stage III melanoma. OpACIN (NCT02437279) was a phase Ib trial that randomized 20 patients with stage IIIB and IIIC melanoma with palpable nodes to receive four cycles of adjuvant ipilimumab $3 \mathrm{mg} / \mathrm{kg}$ plus nivolumab $1 \mathrm{mg} / \mathrm{kg}$ every 3 weeks, or two cycles of neoadjuvant therapy followed by two cycles of adjuvant ipilimumab $3 \mathrm{mg} / \mathrm{kg}$ plus nivolumab $1 \mathrm{mg} / \mathrm{kg}$ every 3 weeks [23]. After a mean follow-up of 36 months, the estimated RFS at 3 years for the neoadjuvant arm was $80 \%$, versus $60 \%$ for the adjuvant arm [24]. This survival benefit with neoadjuvant-only therapy came together with an increased expansion of tumor-resident T-cell receptor (TCR) clones. In the OpACIN trial, none of the seven patients who achieved a pathologic response upon neoadjuvant therapy relapsed (median follow-up of 48.0 months) [24].

The subsequent OpACIN-neo phase II trial (NCT02977052) evaluated three different schedules of neoadjuvant ipilimumab plus nivolumab. Overall, 86 patients were randomized $(1: 1: 1)$ to receive two cycles of ipilimumab $3 \mathrm{mg} / \mathrm{kg}$ plus nivolumab $1 \mathrm{mg} / \mathrm{kg}$ every 3 weeks ( $\operatorname{arm~A}, n=30$ ), or two cycles of ipilimumab $1 \mathrm{mg} / \mathrm{kg}$ plus nivolumab $3 \mathrm{mg} / \mathrm{kg}$ every 3 weeks ( $\operatorname{arm~B}, n=30$ ), or two cycles of ipilimumab $3 \mathrm{mg} / \mathrm{kg}$ every 3 weeks followed immediately by two cycles of nivolumab $3 \mathrm{mg} / \mathrm{kg}$ every 2 weeks ( $\operatorname{arm} \mathrm{C}, n=26$ ). Notably, arm $\mathrm{C}$ was closed earlier due to toxicity on advice from the Data and Safety Monitoring Board (DSMB). After a median follow-up of 24.6 months, the median RFS was not achieved in any arms of the OpACIN-neo trial. The estimated 2-year RFS was $84 \%$ for all patients; $90 \%$ for arm A, $78 \%$ for arm B, and $83 \%$ for $\operatorname{arm} \mathrm{C}[24,25]$.

The OpACIN-neo study includes an extension cohort, aiming to confirm the pathologic response rate and safety of neoadjuvant combination therapy according to the arm $\mathrm{B}$ schedule, and to test response-driven subsequent therapy [26]. In the PRADO cohort, patients with measurable clinical stage III melanoma received two cycles of ipilimumab plus nivolumab, after marker placement in the index lymph node (ILN), which is the largest lymph node. Patients achieving major pCR in the ILN did not undergo lymph node dissection (TLND), while patients with pathological partial response (pPR; $>10$ to $\leq 50 \%$ viable tumor cells) underwent TLND and those with no pCR underwent TLND and adjuvant nivolumab or targeted therapy for 52 weeks \pm radiotherapy. Preliminary data from this expansion cohort suggest that combined immunotherapy leads to a high rate of major pCR (61\%) and that TLND can be omitted in the majority of these patients, with reduced surgical morbidity reducing surgical morbidity. However, longer follow-up is needed to report safety and RFS among patients with major pCR not undergoing TLND.

Neoadjuvant combination of ipilimumab plus nivolumab has been compared with nivolumab monotherapy in a randomized phase II trial (NCT02519322) [27]. In that trial, 23 patients with stage IIIB and IIIC melanoma were randomized to receive neoadjuvant nivolumab $3 \mathrm{mg} / \mathrm{kg}$ every 2 weeks for up to four doses $(n=12)$, or combined ipilimumab $3 \mathrm{mg} / \mathrm{kg}$ and nivolumab $1 \mathrm{mg} / \mathrm{kg}$ every 3 weeks for up to three doses $(n=11)$. The trial design of that study planned to enroll a total of 40 patients, however the trial was stopped early by the DSMB based on the observation of disease progression preventing surgical resection during nivolumab monotherapy (17\%), as well as high rates of serious immune-related adverse events (irAEs) in the combination arm (73\%). With the limits related to the early closure of the trial and lack of 
statistical significance of available data, combined immunotherapy was associated with higher pCR rates and improved RFS, DMFS, and OS (especially among patients reaching pCR) [27].

Neoadjuvant targeted therapy with dabrafenib and trametinib has been investigated in the context of two phase II clinical trials [28, 29]. The first trial (NCT02231775) included patients with locally advanced clinical stage III (i.e. with at least one palpable lymph node metastasis or in-transit metastasis) or oligometastatic stage IV (i.e. fewer than four sites of metastases) BRAF V600E/K-mutated melanoma $(n=21)$ [28]. Patients were randomly assigned $(1: 2)$ to either upfront surgery followed by standard adjuvant therapy, or neoadjuvant ( 8 weeks) plus adjuvant (up to 44 weeks) dabrafenib and trametinib. Notably, the trial was stopped earlier after a prespecified interim safety analysis revealed significantly longer event-free survival (EFS) with neoadjuvant plus adjuvant dabrafenib and trametinib than with standard of care. After a median follow-up of 18.6 months, EFS was higher among patients receiving neoadjuvant treatment compared with patients in the control group (median EFS, 19.7 and 2.9 months in the neoadjuvant and control groups, respectively). No significant differences were observed regarding toxicities among the two treatment groups.

The NeoCombi trial (NCT01972347) was a single-arm, phase II trial aiming to investigate the proportion of patients with pCR after neoadjuvant dabrafenib plus trametinib [29]. This trial enrolled 35 patients with clinically evident stage IIIB-C BRAF V600-mutated melanoma who received 12 weeks of targeted therapy before surgery. The median follow-up was 27 months. At the time of resection, $86 \%$ of patients had a disease response, with $46 \%$ having complete response and $40 \%$ having partial response; no patients experienced disease progression. All patients achieved a pCR, with $49 \%$ of patients reaching a complete $\mathrm{pCR}$. The toxicity profile of dabrafenib and trametinib was similar to that observed in the adjuvant and metastatic settings, and the surgical complications rate was similar to historical data.

Together, evidence supports the use of both neoadjuvant immunotherapy and targeted therapy for melanoma. Immunotherapy surely holds more promise in this setting due to increased tumor-associated antigen exposure during the period in which the major tumor mass is present (i.e. before surgery) and subsequent enhanced and broader T-cell activation promoted by immune checkpoint inhibitors [20]. Moreover, neoadjuvant immunotherapy has been correlated with the persistence of circulating tumor-specific CD8+ T cells in the blood mouse models, acting as memory immune cells. This can be particularly relevant if confirmed in the clinical setting, as it suggests that neoadjuvant treatment might provide long-lasting protection against tumor reappearance [20].

\section{Circulating Biomarkers}

Predictive and prognostic biomarkers were first identified in advanced and metastatic melanoma patients undergoing systemic treatment. The most important clinical prognostic factors that could predict the outcome of melanoma patients include histologic features of primary melanoma (Breslow thickness, presence of ulceration, mitotic rate); the site and number of distant metastases (i.e. tumor burden) in patients with metastatic disease; the number of positive lymph nodes and the burden of nodal involvement in patients with stage III disease; and the serum lactate dehydrogenase (LDH) level [30].

Blood tests are indeed feasible, non-invasive tools providing several potential predictive and prognostic circulating biomarkers. As an example, a complete blood count contains multiple easy-to-use biomarkers, including absolute and relative counts of white blood cell subpopulations (e.g. neutrophil, lymphocyte, eosinophil), and neutrophilto-lymphocyte ratio (NLR), which demonstrated correlation with outcomes during immunotherapy [31]. Promising biomarkers also include antibodies against NY-ESO 1 [32], circulating T-regulatory cells (Tregs) and myeloid-derived suppressor cell (MDSC) counts [33]. Other quantitative circulating biomarkers, which are released upon rapid and massive proliferation of tumor cells, reflect higher aggressiveness and tumor burden [34, 35]. High levels of serum LDH and S100B have demonstrated correlation with poor prognosis in not only patients with metastatic melanoma [36] but also in those with high-risk resected disease [37] (Table 1). However, the role of LDH and S100B in the adjuvant setting has not been clearly defined and neither of these biomarkers are to be considered mandatory for the prognostic evaluation of patients with stage III resected disease [37-39].

Few biomarkers have been specifically evaluated for the prediction of disease relapse in patients undergoing surgery for stage II and III melanoma. A retrospective study investigated the role of circulating biomarkers (serum LDH, melanoma-inhibiting activity [MIA], and calcium binding protein $\mathrm{S} 100 \mathrm{~B}$ ) and their dynamic changes over time, with OS in melanoma patients treated with adjuvant interferon (IFN)- $\alpha 2 b$ [40]. In this analysis, all of the above biomarkers were significantly related to survival outcomes, and the dynamic change of LDH was the most significant predictor of OS. However, adjuvant IFN therapy is no longer the standard of care in high risk, resected melanoma since it has been replaced, almost worldwide, by more effective drugs.

Cell-free DNA (cfDNA) and circulating tumor DNA (ctDNA) have emerged as promising biomarkers in several types of tumors [41, 42]. Small amounts of cfDNA 


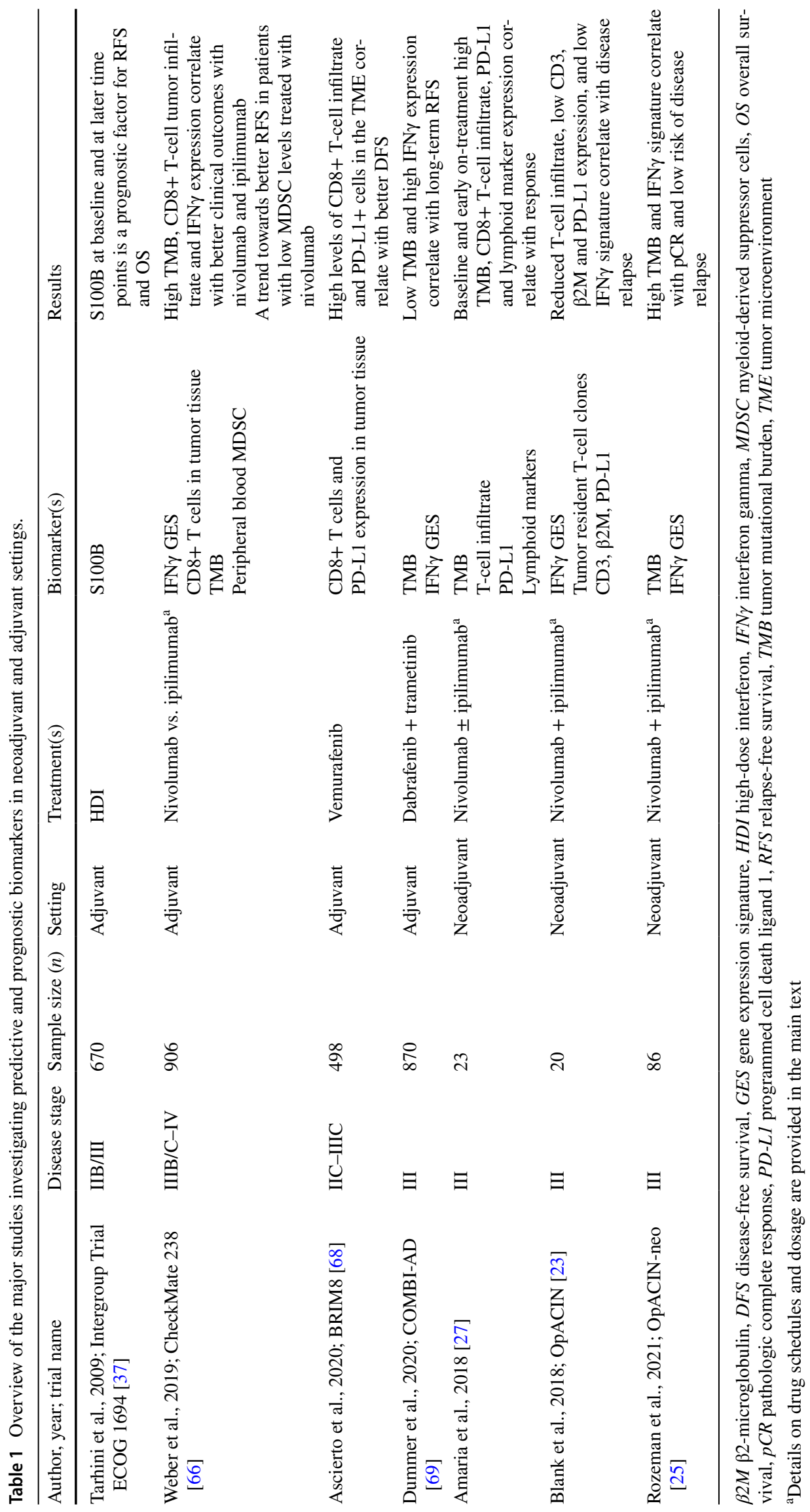


can be detected in human plasma at very low concentrations under physiological conditions. The cfDNA amount is significantly higher in patients with cancer and is also higher in patients with advanced-stage tumors compared with earlier-stage tumors, due to the release of DNA fragments by tumor cells in the blood stream, namely ctDNA. In patients with metastatic melanoma receiving targeted therapy, on-treatment monitoring of plasma BRAF p. V600E cfDNA concentrations have shown a correlation with survival outcomes [42]. Specifically, undetectable cfDNA p.V600E before and during treatment was demonstrated to correlate with a favorable prognosis [42]. In a recently published clinical validation study, baseline and on-treatment ctDNA was evaluated in BRAF-mutated metastatic melanoma patients receiving dabrafenib plus trametinib in two clinical trials, the COMBI-d and the COMBI-MB [43]. In that study, elevated baseline BRAF V600-positive ctDNA concentration was associated with worse survival outcomes, regardless of clinical characteristics and LDH levels. Moreover, undetectable ctDNA after the first 4 weeks of treatment correlated with better PFS and OS, especially among patients with elevated baseline LDH levels [43].

More recent studies demonstrated that the presence of ctDNA in the postoperative setting predicts disease relapse in patients with stage II and III melanoma [44], and detectable preoperative ctDNA is predictive of outcome in this population of patients $[45,46]$. According to these results, detection of ctDNA allows the identification of patients at higher risk of early disease relapse and worse survival. Moreover, ctDNA is a minimally invasive and relatively inexpensive blood test analysis, which can be particularly useful in the stratification of patients in the routine clinical setting. Digital polymerase chain reaction (dPCR) methodology is one of the most convenient methods in this setting, given its limited costs, high specificity, and accuracy. However, the dPCR approach lacks sensitivity since it covers BRAF, NRAS, and TERT mutations, which together occur in more than $80 \%$ of cutaneous melanomas [47]. Other methods of ctDNA detection, such as next-generation sequencing (NGS), can detect genome-wide DNA variation with the aid of multiple targeted gene panels, with lower costs compared with whole genome sequencing (WGS) and whole exome sequencing (WES). However, sequencing costs might significantly increase in the case of rare allele detection at a low frequency, since a very high coverage (> $10,000 \times$ ) is required [48]. Longitudinal sampling of ctDNA after surgery could also be used during adjuvant treatment to detect disease relapse before radiological evidence. Intrinsic limitations of ctDNA liquid biopsies include low sensibility and accuracy, mainly related to low signal-to-noise ratio and ctDNA short half-life, together with the lack of standardization of this technique across different studies.
S100A8/A9 (also known as MRP8 and MRP14, respectively) are $\mathrm{Ca}^{2+}$ binding proteins belonging to the $\mathrm{S} 100$ family. They are members of the damage-associated molecular pattern (DAMP), production of which is upregulated upon cell damage, inflammation, and ultraviolet (UV) radiation [49]. These heterodimers are also reported to be increased in the tissue and serum of patients with solid tumors [50]. S100A8/A9 promote melanoma cells metastases [51] and contribute to the maintenance of immune-suppressive tumor microenvironment (TME) [52, 53], through MDSC recruitment and suppression of CD8+ T-cell activation, thus favoring disease progression and reduced response to systemic immunotherapy. An analysis of gene and protein expression of the TME-derived protein S100A8/A9 in the tissue of metastatic melanoma patients revealed that S100A8/ A9 gene expression was increased in metastases compared with primary melanomas [54]. Interestingly, there was a significantly higher rate of S100A8/A9-expressing cells in metastasizing primary melanomas compared with nonmetastasizing primary melanomas, and also in metastatic melanoma tissue sections of short-term survivors compared with long-term survivors [54]. Moreover, elevated S100A8/ A9 serum levels were significantly associated with impaired survival in two independent cohorts of patients treated with pembrolizumab [54].

Elevated baseline LDH and S100B levels are relevant serum biomarkers in patients with metastatic melanoma receiving targeted therapy [55]. The repeated assessment of $\mathrm{LDH}$ and S100B levels before and during treatment demonstrated correlation with disease-specific survival in a retrospective study of 191 melanoma patients treated with BRAF inhibitors with or without MEK inhibitors [55]. Moreover, S100B showed a strong correlation with disease response, and the elevation of S100B levels predicted radiologic disease progression as early as 8 weeks before the evidence of radiologic disease progression [55].

\section{Tumor Microenvironment}

Deeply linked with the analysis of circulating biomarkers, the evaluation of TME can provide potential predictors for disease relapse and response to systemic treatments (Fig. 1). There is a complex interplay among tumor cells, immune cells and the TME, not only during immunotherapy but also during targeted therapy. Recent evidence suggests that BRAF and MEK inhibitors deeply interact with the host immune response to melanoma by enhancing the expression of melanoma differentiation antigens, reducing levels of immunosuppressive cytokines in the TME, and impairing CD8+ T-cell-mediated responses and cytotoxicity [56]. The presence of $B R A F$ mutation modulates $\mathrm{CD} 8+\mathrm{T}$-cell infiltration and PD-L1 expression on immune cells [57, 58]. Indeed, 
growing evidence supports that the therapeutic efficacy of BRAF and MEK inhibitors, beside its molecular mechanism of action, also relies on the priming of tumor-host interactions through the modulation of melanoma-associated antigen expression, the increase of PD-1/PD-L1 expression, and a decrease in immunosuppressive cytokines in the TME, thus enhancing the immune response against tumor cells [59]. Analysis of tumor biopsies from 213 patients treated with $\mathrm{BRAF} \pm$ MEK inhibitors within the Italian Melanoma Intergroup showed that both density and spatial distribution of key immune cells in the TMN could contribute to treatment response [60].

Studies of patients with metastatic disease have identified several biomarkers, both in peripheral blood and in the context of tumor tissue. In patients with metastatic melanoma, the presence of CD8+ T lymphocytes and programmed death ligand 1 (PD-L1)-positive immune cells, associated with a decrease in Tregs in the TME, has shown to predict the outcomes of treatment with checkpoint inhibitors [61, 62].

High tumor mutational burden (TMB) was associated with long-term clinical benefit in patients with metastatic melanoma receiving ipilimumab [63], and this evidence was further confirmed in patients treated with nivolumab [64]. The combination of higher TMB and T-cell-inflamed TME have been identified as crucial factors for response during anti-PD1 immunotherapy for metastatic disease [65]. Similar results were reported in patients receiving adjuvant nivolumab in the CheckMate-238 trial [66]. In this exploratory analysis, biomarkers measured at baseline (pretreatment) were IFN $\gamma$ gene expression profile (GEP) signature and $\mathrm{CD} 8+\mathrm{T}$ cells in tumor tissue, TMB, and MDSC in peripheral blood. Patients with high TMB, high levels of CD8+ T-cell tumor infiltration and IFN $\gamma$ expression showed better clinical outcomes (both with nivolumab and ipilimumab) compared with patients with below median TMB, low CD8+ T-cell infiltration and IFN $\gamma$ expression; a trend towards better RFS was observed in patients receiving nivolumab with lower peripheral MDSC levels [66] (Table 1).

A biomarker analysis of the randomized phase III COMBI-i trial (NCT02967692) specifically addressed the role of TMB and gene expression signature (GES) in a cohort of patients receiving the anti-PD-1 antibody spartalizumab in combination with the BRAF inhibitor dabrafenib and MEK inhibitor trametinib $(n=27)$ [67]. In this study, patients with early disease progression more frequently had low baseline TMB and T-cell-inflamed GES levels, and showed high baseline ctDNA levels. Moreover, on-treatment increases of T-cell-inflamed GES levels, together with decreases in MAPK activity score and cell cycle GES levels, were reported, suggesting that combined treatment has an early impact on both tumor cells and the TME. Results from this analysis also suggest that the presence of immunosuppressive components (e.g. M2 macrophages) within the TME might preclude an adequate response to treatment, even in patients showing a favorable biomarker profile (e.g. with high levels of T-cell-inflamed or IFN $\gamma$ ) [67].

To date, there is limited evidence on the role of TME components in adjuvant melanoma, and most components are derived from the major clinical trials of adjuvant targeted therapy and immunotherapy in patients with stage II/ III disease [66, 68, 69]. A retrospective exploratory analysis of patients with resected stage IIC-IIIC BRAF V600-mutant melanoma treated with adjuvant vemurafenib in the BRIM8 trial showed that the presence of CD8+ T cells and PD-L1 expression on tumor-associated immune cells has a positive correlation with disease-free survival (DFS) [68]. Higher levels of CD8+ T-cell infiltrate and the presence of PD-L1+ cells in the TME correlated with better DFS, even in patients receiving placebo, suggesting that these two parameters have a positive prognostic impact in this subset of patients [68] (Table 1).

The most relevant data on prognostic biomarkers for adjuvant targeted therapy in patients with cutaneous melanoma derive from an exploratory biomarker analysis of the randomized phase III COMBI-AD trial [69] (Table 1). This was the first large-scale analysis with comprehensive DNA sequencing and GES analysis evaluating the role of TMB and T-cell-inflamed GES in patients receiving adjuvant targeted therapy. Results from this analysis showed a strong prognostic association between high IFN $\gamma$ GES and prolonged RFS in patients in both the placebo and targeted therapy groups. TMB had a prognostic role in the placebo group but not in the dabrafenib plus trametinib group. Patients with a low TMB had a greater benefit from adjuvant targeted therapy, while patients with high TMB had less benefit from adjuvant treatment, especially if they had a low IFN $\gamma$ signature. Similar results had already been demonstrated in The Cancer Genome Atlas (TCGA) dataset, in which mutational load was not correlated with T-helper 1 or IFN $\gamma$ signature, but had an independent prognostic value [70]. Previous studies have already shown that immune GESs were associated with favorable clinical outcomes after treatment with MAPK inhibitors [71]. High TMB might have a negative effect on targeted therapy, potentially related to an increased number of escape mechanisms derived from genetic heterogeneity. Data from early and advanced melanoma patients treated with immunotherapy confirmed that patients with low TMB and low immune gene expression had a poor response to treatment [71]. Conversely, targeted therapy in the COMBI-AD trial provided RFS benefit in the subgroup of patients with low TMB and low IFN $\gamma$ GES, who would not be expected to derive significant benefit from adjuvant immunotherapy [69]. The population with the greatest clinical benefit from adjuvant targeted therapy in the 
COMBI-AD trial was the low TMB and high IFN $\gamma$ signature subgroup [69]. A TCGA analysis of patients in this subgroup showed a correlation with the toll-like receptor (TLR) and Fas signaling gene sets, together with lower expression of immune checkpoint genes (PD-L1, PD-L2, LAG3, IDO1, and TIGIT) compared with the high TMB and high IFN $\gamma$ signature subgroups. Similarly, a subgroup analysis from the CheckMate 067 trial showed that tumors with low TMB and high immune GES seemed to derive the greatest benefit from combination immunotherapy with anti-CTLA4 and anti-PD1 [72]. Together, these results suggest that combination therapy (either with targeted or immunotherapy) might have a significant role in this specific subgroup of patients.

Similar results have been observed in the context of neoadjuvant treatment. Biomarker analysis of a phase II trial (NCT02519322) comparing neoadjuvant nivolumab with a combination of nivolumab and ipilimumab, showed that responders had not only high TMB but also higher CD8+ T-cell infiltrate, tumor cell PD-L1 expression, and expression of lymphoid markers (Granzyme B, CD4, FoxP3, CD20, and PD-1) at baseline and at early on-treatment biopsies [27]. Additional immune markers correlated with response were expression of CD45RO, $\beta 2$-microglobulin, and T-cell (CD3, CD8) and B-cell markers (CD19, CD20) [27].

The most relevant data on biomarkers in the neoadjuvant setting come from exploratory analysis of the OpACIN and OpACIN-neo trials [25]. Baseline IFN $\gamma$ gene signature expression was associated with absence of relapse in the OpACIN study [23] and with higher pCR rates and low risk of relapse in the OpACIN-neo trial [25]. Baseline TMB was strongly associated with EFS, with an estimated 2-year EFS of $93 \%$ for patients with TMB greater than the median, versus $59 \%$ for patients with TMB less than the median [25]. Even this analysis confirmed that the combination of IFN $\gamma$ signature and TMB allows the identification of patients more likely to respond to neoadjuvant treatment, specifically those with high IFN $\gamma$ signature and high TMB [25]. Patients responding to neoadjuvant treatment also showed higher levels of all immune cell populations in tumor tissue, as well as upregulation of proliferation and signaling of several immune pathways, while non-responders showed upregulation of angiogenesis and epithelial-to-mesenchymal transition gene sets [25] (Table 1).

\section{Gene Expression Profile and Genetic Signatures}

GEP signatures have been shown to be promising prognostic tools in several solid tumors and also in cutaneous melanoma [73]. Molecular characterization of tumors, in addition to traditional staging systems, can be used to improve prognostic accuracy and risk stratification. A 31-GEP test that dichotomizes cutaneous melanoma patients as Class 1 (low-risk) or Class 2 (high-risk) patients has been identified, and demonstrated to accurately predict metastatic risk in patients eligible for sentinel node biopsy [74]. This is particularly relevant, considering that approximately two-thirds of melanoma-related deaths in patients originally diagnosed with early-stage disease occur in patients without evidence of disease at sentinel node biopsy (i.e. stage I-II) [2]. On the other hand, a significant proportion of stage III patients, particularly those with only evidence of microscopic disease at sentinel node biopsy, will never experience disease progression [2]. This evidence suggests that a high proportion of patients with biologically aggressive disease are still undetected by current diagnostic techniques [75]. In a further study, this 31-gene GEP was evaluated in an independent cohort of 523 cutaneous melanoma patients [76]. The 5 -year DMFS rate in this study was $93 \%$ for Class 1 patients and $62 \%$ for Class 2 patients (compared with $100 \%$ and $58 \%$,
Fig. 1 Major circulating and tissue biomarkers currently under study in neoadjuvant and adjuvant settings of melanoma. $c f D N A$ cell-free DNA, $c t D N A$ circulating tumor DNA, FAS- $L$ FAS ligand, $I F N \gamma$ interferon gamma, $M D S C$ myeloid-derived suppressor cell, $M H C$ major histocompatibility complex, $N K$ natural killer, $P D-L 1$ programmed cell death ligand 1, TCR T-cell receptor, Treg regulatory $\mathrm{T}$ cell

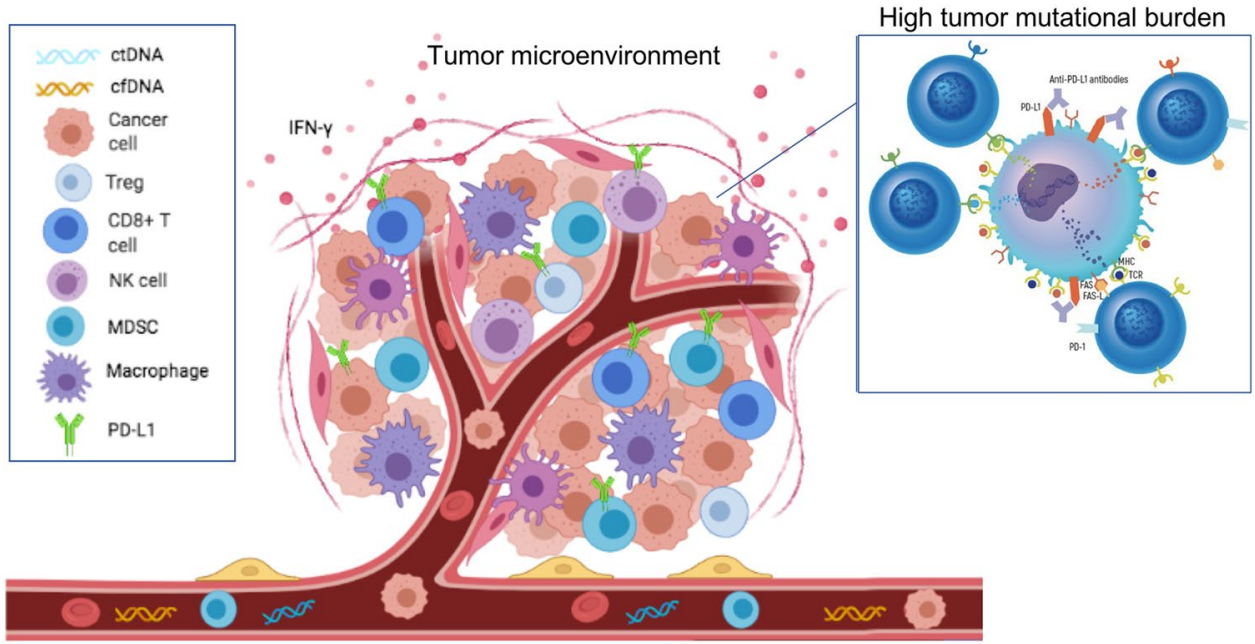


respectively, in the first study), thus confirming the predictive role of 31-gene GEP in this subset of patients [76].

Genetic mutations within tumor cells play an important role in resistance to immunotherapy in metastatic melanoma and could also be implicated in escape mechanisms during adjuvant immunotherapy. Recognized mechanisms involved in resistance to immune checkpoint inhibitors include the activation of the WNT/ $\beta$-catenin signaling pathway, which induces T-cell exclusion [77]; the loss of PTEN, which reduces T-cell expansion and tumor infiltration [78]; mutations in the Janus kinase (JAK1/JAK2) pathway, which impairs IFN $\gamma$ signaling [79]; point mutations, deletions, or loss of heterozygosity ( $\mathrm{LOH}$ ) in $\beta 2$-microglobulin, an essential component of MHC class I antigen presentation [80]; and increased production of transforming growth factor (TGF)- $\beta$, which is involved in the regulation of cell proliferation, differentiation, and survival [81]. Assessment of these parameters in the context of adjuvant immunotherapy would be interesting in order to verify their potential role in primary or acquired resistance, similar to metastatic disease.

\section{Conclusions and Future Perspectives}

Novel therapeutic strategies have significantly improved the prognosis of melanoma. However, the majority of patients will gain only partial and transient benefit from systemic therapy and will eventually progress and die from melanoma. Recent research has improved the knowledge of the factors contributing to the immune escape, with the identification of several potential predictive and prognostic biomarkers. This has translated into the development of prognostic and predictive tools for both early and advanced disease. In the context of adjuvant treatment, biomarkers for a strong adaptive immune response (i.e. TMB alone or in combination with IFN $\gamma$ signature) seem to identify patients with stage III melanoma who derive clinical benefit from both targeted therapy and immune checkpoint inhibitors. Similar results are supported in the neoadjuvant setting and are even more intriguing in the perspective of tailoring treatment according to the rate of pathologic response. Results of clinical trials conducted to date suggest that neoadjuvant therapy resulting in $\mathrm{pCR}$ or near-pCR identified a subgroup of patients with durable responses and extremely good prognosis [82]. Conversely, patients with resistant disease represent an unmet need and should be studied in order to improve treatment selection and outcomes. Clinical trials are currently undergoing to define whether pathological correlates can be used to customize treatment. In the phase III NADINA 2021 trial, patients with stage III melanoma are randomized to upfront surgery (TLND) followed by adjuvant nivolumab (control group) or a neoadjuvant combination of nivolumab and ipilimumab (two courses) followed by TLND (experimental arm). Patients in the experimental arm showing $\mathrm{pCR}$, or near-pCR (i.e. $\leq 50 \%$ vital tumor cells), will start follow-up, while those not achieving pCR will receive adjuvant treatment according to BRAF mutational status (i.e. nivolumab in BRAF wild-type patients, or combined dabrafenib and trametinib in BRAF V600mutant patients). The DONIMI trial (NCT04133948) is a phase Ib study evaluating the combination of the histone deacetylase (HDAC) inhibitor domatinostat, plus nivolumab versus nivolumab monotherapy in IFN $\gamma$ signature-high patients, and domatinostat plus nivolumab or plus nivolumab and ipilimumab in IFN $\gamma$ signature-low patients with de novo or recurrent macroscopic stage III cutaneous or unknown primary melanoma. The aim of this study was to increase patients' susceptibility to immunotherapy, specifically in patients with IFN $\gamma$ signature-low, by adding domatinostat to immune checkpoint inhibitors. Biomarkers are also under investigation to anticipate disease relapse diagnosis in earlier-stage melanoma. The DETECTION study evaluated the role of periodic ctDNA monitoring in patients with stage IIB/C melanoma with BRAF, NRAS, or TERT promoter mutation. At the time of ctDNA detection, patients are randomized to receive the investigators' choice of systemic treatment at the time of radiologically confirmed disease relapse (arm A), or treatment with nivolumab starting at the time of molecular recurrence. Although promising, available results still need to be implemented and prospectively confirmed to be finally employed in everyday clinical practice. Ongoing clinical trials will improve the knowledge in this field, with the aim of developing more effective and less toxic treatments, and further improve the prognosis of patients with melanoma.

\section{Declarations}

Funding This manuscript was funded by the Fondazione AIRC "Programma di ricerca 5 per Mille 2018"--ID\#21073. Open access funding was provided by UniversitÃ degli Studi di Perugia within the CRUICARE Agreement.

Conflicts of interest/Competing interest Alice Indini, Fausto Roila, Francesco Grossi, Daniela Massi, and Mario Mandalà declare that this research was conducted in the absence of any commercial or financial relationships that could be construed as a potential conflict of interest.

Data availability statement Not applicable.

Author contributions Conceptualization: AI, MM. Methodology: AI, DM, MM. Supervision: FG, FR, MM. Writing-original draft: AI, MM. Writing - review and editing: FG, DM, FR, MM.

Code availability Not applicable.

Ethics approval Not applicable. 
Consent to participate Not applicable.

Consent for publication Not applicable.

Open Access This article is licensed under a Creative Commons Attribution-NonCommercial 4.0 International License, which permits any non-commercial use, sharing, adaptation, distribution and reproduction in any medium or format, as long as you give appropriate credit to the original author(s) and the source, provide a link to the Creative Commons licence, and indicate if changes were made. The images or other third party material in this article are included in the article's Creative Commons licence, unless indicated otherwise in a credit line to the material. If material is not included in the article's Creative Commons licence and your intended use is not permitted by statutory regulation or exceeds the permitted use, you will need to obtain permission directly from the copyright holder. To view a copy of this licence, visit http://creativecommons.org/licenses/by-nc/4.0/.

\section{References}

1. Siegel RL, Miller KD, Jemal A. Cancer statistics, 2017. CA Cancer J Clin. 2017;67:7-30.

2. Gershenwald JE, Scolyer RA, Hess KR, Sondak VK, Long GV, Ross MI, et al. Melanoma staging: Evidence-based changes in the American Joint Committee on Cancer Eighth Edition cancer staging manual. CA Cancer J Clin. 2017;67(6):472-92. https:// doi.org/10.3322/caac. 21409 .

3. Amin MB, Edge SB, Greene FL, Byrd DR, Brookland RK, Washington MK, et al editors. AJCC cancer staging manual. 8th ed. New York: Springer; 2016.

4. Hodi FS, O'Day SJ, McDermott DF, Weber RW, Sosman JA, Haanen JB, et al. Improved survival with ipilimumab in patients with metastatic melanoma. N Engl J Med. 2010;363(8):711-23. https://doi.org/10.1056/NEJMoa1003466.

5. Larkin J, Chiarion-Sileni V, Gonzalez R, Grob JJ, Cowey CL, Lao $\mathrm{CD}$, et al. Combined nivolumab and ipilimumab or monotherapy in untreated melanoma. N Engl J Med. 2015;373(1):23-34. https://doi.org/10.1056/NEJMoa1504030.

6. Robert C, Schachter J, Long GV, Arance A, Grob JJ, Mortier L, et al. Pembrolizumab versus ipilimumab in advanced melanoma. N Engl J Med. 2015;372(26):2521-32. https://doi.org/10.1056/ NEJMoa1503093.

7. Larkin J, Ascierto PA, Dréno B, Atkinson V, Liszkay G, Maio M, et al. Combined vemurafenib and cobimetinib in BRAF-mutated melanoma. N Engl J Med. 2014;371:1867-76. https://doi.org/10. 1056/NEJMoa1408868.

8. Long GV, Stroyakovskiy D, Gogas H, Levchenko E, de Braud F, Larkin J, et al. Dabrafenib and trametinib versus dabrafenib and placebo for Val600 BRAF-mutant melanoma: a multicentre, double-blind, phase 3 randomised controlled trial. Lancet. 2015;386(9992):444-51. https://doi.org/10.1016/S0140-6736(15) 60898-4

9. Dummer R, Ascierto PA, Gogas HJ, Arance A, Mandala M, Liszkay $\mathrm{G}$, et al. Encorafenib plus binimetinib versus vemurafenib or encorafenib in patients with BRAF-mutant melanoma (COLUMBUS): a multicentre, open-label, randomised phase 3 trial. Lancet Oncol. 2018;19(5):603-15. https://doi.org/10.1016/S14702045(18)30142-6.

10. Testori AAE, Ribero S, Indini A, Mandalà M. Adjuvant treatment of melanoma: recent developments and future perspectives. Am J Clin Dermatol. 2019;20(6):817-27. https://doi.org/10.1007/ s40257-019-00456-4.
11. Eggermont AM, Chiarion-Sileni V, Grob JJ, Dummer R, Wolchok JD, Schmidt H, et al. Adjuvant ipilimumab versus placebo after complete resection of high-risk stage III melanoma (EORTC 18071): a randomised, double-blind, phase 3 trial. Lancet Oncol. 2015;16(5):522-30. https://doi.org/10.1016/S1470-2045(15) 70122-1.

12. Eggermont AM, Chiarion-Sileni V, Grob JJ, Dummer R, Wolchok JD, Schmidt H, et al. Prolonged survival in stage III melanoma with ipilimumab adjuvant therapy (published erratum appears in N Engl J Med. 2018;379(22):2185). N Engl J Med. 2016;375(19):1845-55. https://doi.org/10.1056/NEJMoa1611299.

13. Weber J, Mandala M, Del Vecchio M, Gogas HJ, Arance $\mathrm{AM}$, Cowey CL, et al. Adjuvant nivolumab versus ipilimumab in resected stage III or IV melanoma. N Engl J Med. 2017;377(19):1824-35. https://doi.org/10.1056/NEJMoa1709030.

14. Ascierto PA, Del Vecchio M, Mandalá M, Gogas H, Arance AM, Dalle S, et al. Adjuvant nivolumab versus ipilimumab in resected stage IIIB-C and stage IV melanoma (CheckMate 238): 4-year results from a multicentre, double-blind, randomised, controlled, phase 3 trial. Lancet Oncol. 2020;21(11):1465-77. https://doi.org/10.1016/S1470-2045(20)30494-0.

15. Eggermont AMM, Blank CU, Mandala M, Long GV, Atkinson VG, Dalle $S$, et al. Adjuvant pembrolizumab versus placebo in resected stage III melanoma. N Engl J Med. 2018;378(19):1789-801. https://doi.org/10.1056/NEJMoa1802 357.

16. Eggermont AMM, Blank CU, Mandala M, Long GV, Atkinson VG, Dalle $S$, et al. Longer follow-up confirms recurrence-free survival benefit of adjuvant pembrolizumab in high-risk stage III melanoma: updated results from the EORTC 1325-MG/KEYNOTE-054 trial. J Clin Oncol. 2020;38(3):3925-36. https://doi. org/10.1200/JCO.20.02110.

17. Long GV, Hauschild A, Santinami M, Mandalà M, Chiarion-Sileni V, Larkin J, et al. Adjuvant dabrafenib plus trametinib in stage III BRAF-mutated melanoma. N Engl J Med. 2017;377(19):1813-23. https://doi.org/10.1056/NEJMoa1708539.

18. Dummer R, Hauschild A, Santinami M, Atkinson V, Mandalà M, Kirkwood JM, et al. Five-year analysis of adjuvant dabrafenib plus trametinib in stage III melanoma. N Engl J Med. 2020;383(12):1139-48. https://doi.org/10.1056/NEJMoa2005493.

19. Eggermont AMM, Robert $\mathrm{C}$, Ribas $\mathrm{A}$. The new era of adjuvant therapies for melanoma. Nat Rev Clin Oncol. 2018;15(9):535-6. https://doi.org/10.1038/s41571-018-0048-5.

20. O'Donnell JS, Hoefsmit EP, Smyth MJ, Blank CU, Teng MWL. The promise of neoadjuvant immunotherapy and surgery for cancer treatment. Clin Cancer Res. 2019;25:5743-51. https://doi.org/ 10.1158/1078-0432.CCR-18-2641.

21. Menzies AM, Amaria RN, Rozeman EA, Huang AC, Tetzlaff MT, van de Wiel BA, et al. Pathological response and survival with neoadjuvant therapy in melanoma: a pooled analysis from the International Neoadjuvant Melanoma Consortium (INMC). Nat Med. 2021;27:301-9. https://doi.org/10.1038/ s41591-020-01188-3.

22. Bloemendal M, van Willigen WW, Bol KF, Boers-Sonderen MJ, Bonenkamp JJ, Werner JEM, et al. Early recurrence in completely resected IIIB and IIIC melanoma warrants restaging prior to adjuvant therapy. Ann Surg Oncol. 2019;26:3945-52. https://doi.org/ 10.1245/s10434-019-07274-2.

23. Blank CU, Rozeman EA, Fanchi LF, Sikorska K, van de Wiel B, Kvistborg P, et al. Neoadjuvant versus adjuvant ipilimumab plus nivolumab in macroscopic stage III melanoma. Nat Med. 2018;24:1655-61. https://doi.org/10.1038/s41591-018-0198-0.

24. Blank CU, Versluis JM, Rozeman EA, Menzies AM, Reijers ILM, Krijgsman O, et al. 36-months and 18-months relapse-free survival after (neo)adjuvant ipilimumab plus nivolumab in macroscopic stage III melanoma patients-update of the OpACIN 
and OpACIN-neo trials. In: Presented at the AACR 2020 Annual Meeting; Session MS.IM02.02.

25. Rozeman EA, Hoefsmit EP, Reijers ILM, Saw RPM, Versluis JM, Krijgsman O, et al. Survival and biomarker analyses from the OpACIN-neo and OpACIN neoadjuvant immunotherapy trials in stage III melanoma. Nat Med. 2021;27:256-63. https://doi.org/ 10.1038/s41591-020-01211-7.

26. Blank CU, Reijers ILM, Pennington T, Versluis JM, Saw RPM, Rozeman EA, et al. First safety and efficacy results of PRADO: a phase II study of personalized response-driven surgery and adjuvant therapy after neoadjuvant ipilimumab (IPI) and nivolumab (NIVO) in resectable stage III melanoma. J Clin Oncol. 2020;38(15 Suppl):10002.

27. Amaria RN, Reddy SM, Tawbi HA, Davies MA, Ross MI, Glitza IC, et al. Neoadjuvant immune checkpoint blockade in high-risk resectable melanoma. Nat Med. 2018;24:1649-54. https://doi.org/ 10.1038/s41591-018-0197-1.

28. Amaria RN, Prieto PA, Tetzlaff MT, Reuben A, Andrews MC, Ross MI, et al. Neoadjuvant plus adjuvant dabrafenib and trametinib versus standard of care in patients with high-risk, surgically resectable melanoma: a single-centre, open-label, randomized, phase 2 trial. Lancet Oncol. 2018;19:181-93.

29. Long GV, Saw RPM, Lo S, Nieweg OE, Shannon KF, Gonzalez $\mathrm{M}$, et al. Neoadjuvant dabrafenib combined with trametinib for resectable, stage IIIB-C, BRAFV600 mutation-positive melanoma (NeoCombi): a single-arm, open-label, single-centre, phase 2 trial. Lancet Oncol. 2019;20:961-71.

30. Garbe C, Peris K, Hauschild A, Middleton M, Bastholt L, Grob JJ, et al. Diagnosis and treatment of melanoma. European consensusbased interdisciplinary guideline-Update 2016. Eur J Cancer. 2016;63:201-17. https://doi.org/10.1016/j.ejca.2016.05.005.

31. Indini A, Di Guardo L, Cimminiello C, Prisciandaro M, Randon G, De Braud F, et al. Developing a score system to predict therapeutic outcomes to anti-PD-1 immunotherapy in metastatic melanoma. Tumori. 2019;105(6):465-73. https://doi.org/10.1177/ 0300891619868009.

32. Fässler M, Diem S, Mangana J, Hasan Ali O, Berner F, Bomze D, et al. Antibodies as biomarker candidates for response and survival to checkpoint inhibitors in melanoma patients. J Immunother Cancer. 2019;7(1):50. https://doi.org/10.1186/s40425-019-0523-2.

33. Martens A, Wistuba-Hamprecht K, Geukes Foppen M, Yuan J, Postow MA, Wong P, et al. Baseline peripheral blood biomarkers associated with clinical outcome of advanced melanoma patients treated with ipilimumab. Clin Cancer Res. 2016;22(12):2908-18. https://doi.org/10.1158/1078-0432.CCR-15-2412.

34. Ghanem G, Loir B, Morandini R, Sales F, Lienard D, Eggermont $\mathrm{A}$, et al. On the release and half-life of $\mathrm{S} 100 \mathrm{~B}$ protein in the peripheral blood of melanoma patients. Int $\mathrm{J}$ Cancer. 2001;94:586-90.

35. Gebhardt C, Lichtenberger R, Utikal J. Biomarker value and pitfalls of serum S100B in the follow-up of high-risk melanoma patients. J Dtsch Dermatol Ges. 2016;14(2):158-64.

36. Hauschild A, Engel G, Brenner W, Glaser R, Monig H, Henze E, et al. S100B protein detection in serum is a significant prognostic factor in metastatic melanoma. Oncology. 1999;56:338-44.

37. Tarhini AA, Stuckert J, Lee S, Sander C, Kirkwood JM. Prognostic significance of serum S100B protein in high-risk surgically resected melanoma patients participating in Intergroup Trial ECOG 1694. J Clin Oncol. 2009;27:38-44.

38. Wevers KP, Kruijff S, Speijers MJ, Bastiaannet E, Muller Kobold AC, Hoekstra HJ. S-100B: a stronger prognostic biomarker than LDH in stage IIIB-C melanoma. Ann Surg Oncol. 2013;20:2772-9.

39. Egberts F, Hitschler WN, Weichenthal M, Hauschild A. Prospective monitoring of adjuvant treatment in high-risk melanoma patients: lactate dehydrogenase and protein S-100B as indicators of relapse. Melanoma Res. 2009;19:31-5.

40. Irurzun-Arana I, Asín-Prieto E, Martín-Algarra S, Trocóniz IF. Predicting circulating biomarker response and its impact on the survival of advanced melanoma patients treated with adjuvant therapy. Sci Rep. 2020;10(1):7478. https://doi.org/10.1038/ s41598-020-63441-6.

41. Coco S, Alama A, Vanni I, Fontana V, Genova C, Dal Bello MG, et al. Circulating cell-free DNA and circulating tumor cells as prognostic and predictive biomarkers in advanced non-small cell lung cancer patients treated with first-line chemotherapy. Int J Mol Sci. 2017;18:1035. https://doi.org/10.3390/ijms18051035.

42. Kozak K, Kowalik A, Gos A, Wasag B, Lugowska I, Jurkowska $\mathrm{M}$, et al. Cell-free DNA BRAF V600E measurements during BRAF inhibitor therapy of metastatic melanoma: long-term analysis. Tumori. 2020. https://doi.org/10.1177/0300891619900928.

43. Syeda MM, Wiggis JM, Corless BC, Long GV, Flaherty KT, Schadendorf D, et al. Circulating tumour DNA in patients with advanced melanoma treated with dabrafenib or dabrafenib plus trametinib: a clinical validation study. Lancet Oncol. 2021;22:370-80.

44. Tan L, Sandhu S, Lee RJ, Li J, Callahan J, Ftouni S, et al. Prediction and monitoring of relapse in stage III melanoma using circulating tumor DNA. Ann Oncol. 2019;30(5):804-14. https:// doi.org/10.1093/annonc/mdzH.

45. Lee RJ, Gremel G, Marshall A, Myers KA, Fisher N, Dunn JA, et al. Circulating tumor DNA predicts survival in patients with resected high-risk stage II/III melanoma. Ann Oncol. 2018;29(2):490-6. https://doi.org/10.1093/annonc/mdx717.

46. Lee JH, Saw RP, Thompson JF, Lo S, Spillane AJ, Shannon KF, et al. Pre-operative ctDNA predicts survival in high-risk stage III cutaneous melanoma patients. Ann Oncol. 2019;30:815-22. https://doi.org/10.1093/annonc/mdz075.

47. Marczynski GT, Laus AC, Dos Reis MB, Reis RM, Vazquez VL. Circulating tumor DNA (ctDNA) detection is associated with shorter progression-free survival in advanced melanoma patients. Sci Rep. 2020;10:18682. https://doi.org/10.1038/ s41598-020-75792-1.

48. Gebhardt C, Nemeth J, Angel P, Hess J. S100A8 and S100A9 in inflammation and cancer. Biochem Pharmacol. 2006;72(11):1622-31.

49. Chen $\mathrm{M}$, Zhao H. Next-generation sequencing in liquid biopsy: cancer screening and early detection. Hum Genom. 2019;13:34. https://doi.org/10.1186/s40246-019-0220-8.

50. Hermani A, Hess J, De Servi B, Medunjanin S, Grobholz R, Trojan L, et al. Calcium-binding proteins S100A8 and S100A9 as novel diagnostic markers in human prostate cancer. Clin Cancer Res. 2005;11(14):5146-52.

51. Hibino T, Sakaguchi M, Miyamoto S, Yamamoto M, Motoyama A, Hosoi J, et al. S100A9 is a novel ligand of EMMPRIN that promotes melanoma metastasis. Cancer Res. 2013;73(1):172-83.

52. Sinha P, Okoro C, Foell D, Freeze HH, Ostrand-Rosenberg S, Srikrishna G, et al. Proinflammatory S100 proteins regulate the accumulation of myeloid derived suppressor cells. J Immunol. 2008;181(7):4666-75.

53. Cheng P, Corzo CA, Luetteke N, Yu B, Nagaraj S, Bui MM, et al. Inhibition of dendritic cell differentiation and accumulation of myeloid-derived suppressor cells in cancer is regulated by S100A9 protein. J Exp Med. 2008;205(10):2235-49.

54. Wagner NB, Weide B, Gries M, Reith M, Tarnanidis K, Schuermans V, et al. Tumor microenvironment-derived S100A8/ A9 is a novel prognostic biomarker for advanced melanoma patients and during immunotherapy with anti-PD-1 antibodies. J Immunother Cancer. 2019;7(1):343. https://doi.org/10.1186/ s40425-019-0828-1. 
55. Gassenmaier M, Lenders MM, Forschner A, Leiter U, Weide B, Garbe C, et al. Serum S100B and LDH at baseline and during therapy predict the outcome of metastatic melanoma patients treated with BRAF inhibitors. Target Oncol. 2021;16:197-205. https://doi.org/10.1007/s11523-021-00792-8.

56. Kelley MC. Immune responses to BRAF-targeted therapy in melanoma: is targeted therapy immunotherapy? Crit Rev Oncog. 2016;21(1-2):83-91. https://doi.org/10.1615/CritRevOncog. 2016017150 .

57. Wilmott JS, Haydu LE, Menzies AM, Lum T, Hyman J, Thompson JF, et al. Dynamics of chemokine, cytokine, and growth factor serum levels in BRAF-mutant melanoma patients during BRAF inhibitor treatment. J Immunol. 2014;192(5):2505-13. https://doi. org/10.4049/jimmunol.1302616.

58. Ebert PJR, Cheung J, Yang Y, McNamara E, Hong R, Moskalenko $\mathrm{M}$, et al. MAP kinase inhibition promotes $\mathrm{T}$ cell and anti-tumor activity in combination with PD-L1 checkpoint blockade. Immunity. 2016;44(3):609-21. https://doi.org/10.1016/j.immuni.2016. 01.024 .

59. Mandalà M, De Logu F, Merelli B, Nassini R, Massi D. Immunomodulating property of MAPK inhibitors: from translational knowledge to clinical implementation. Lab Invest. 2017;97(2):166-75. https://doi.org/10.1038/labinvest.2016.132.

60. Massi D, Rulli E, Cossa M, Valeri B, Rodolfo M, Merelli B, et al. The density and spatial tissue distribution of CD8+ and CD163+ immune cells predict response and outcome in melanoma patients receiving MAPK inhibitors. J Immunother Cancer. 2019;7(1):308. https://doi.org/10.1186/s40425-019-0797-4.

61. Ouwerkerk W, van den Berg M, van der Niet S, Limpens J, Luiten RM. Biomarkers, measured during therapy, for response of melanoma patients to immune checkpoint inhibitors: a systematic review. Melanoma Res. 2019;29:453-64. https://doi.org/10.1097/ CMR.0000000000000589.

62. Jessurun CAC, Vos JAM, Limpens J, Luiten RM. Biomarkers for response of melanoma patients to immune checkpoint inhibitors: a systematic review. Front Oncol. 2017;7:233. https://doi.org/10. 3389/fonc.2017.00233.

63. Snyder A, Makarov V, Merghoub T, Yuan J, Zaretsky JM, Desrichard A, et al. Genetic basis for clinical response to CTLA-4 blockade in melanoma. N Engl J Med. 2014;371:2189-99.

64. Hugo W, Zaretsky JM, Sun L, Song C, Moreno BH, Hu-Lieskovan $\mathrm{S}$, et al. Genomic and transcriptomic features of resposne to antiPD-1 therapy in metastatic melanoma. Cell. 2016;165:35-44.

65. Ribas A, Robert C, Schachter J, Long GV, Arance A, Carlino MS, et al. Tumor mutational burden (TMB), T cell-inflamed gene expression profile (GEP) and PD-L1 are independently associated with response to pembrolizumab (Pembro) in patients with advanced melanoma in the KEYNOTE (KN)-006 study [abstract]. Cancer Res. 2019;79(Suppl):4217.

66. Weber JS, Del Vecchio M, Mandala M, Gogas H, Arance A, Dalle $\mathrm{S}$, et al. Adjuvant nivolumab (NIVO) versus ipilimumab (IPI) in resected stage III/IV melanoma: 3-year efficacy and biomarker results from the phase III CheckMate 238 trial [abstract]. Ann Oncol. 2019;30(Suppl 5):1310O.

67. Dummer R, Lebbé C, Atkinson V, Mandalà M, Nathan PD, Arance A, et al. Combined PD-1, BRAF and MEK inhibition in advanced BRAF-mutant melanoma: safety run-in and biomarker cohorts of COMBI-i. Nat Med. 2020;26(10):1557-63. https://doi. org/10.1038/s41591-020-1082-2.

68. Ascierto PA, Lewis KD, Di Giacomo AM, Demidov L, Mandalà M, Bondarenko I, et al. Prognostic impact of baseline tumour immune infiltrate on disease-free survival in patients with completely resected, BRAFv600 mutation-positive melanoma receiving adjuvant vemurafenib. Ann Oncol. 2020;31(1):153-9. https://doi.org/10.1016/j.annonc.2019.10.002.

69. Dummer R, Brase JC, Garrett J, Campbell CD, Gasal E, Squires $\mathrm{M}$, et al. Adjuvant dabrafenib plus trametinib versus placebo in patients with resected, BRAFV600-mutant, stage III melanoma (COMBI-AD): exploratory biomarker analyses from a randomised, phase 3 trial. Lancet Oncol. 2020;21(3):358-72. https:// doi.org/10.1016/S1470-2045(20)30062-0.

70. Danilova L, Wang H, Sunshine J, Kaunitz GJ, Cottrell TR, Xu H, et al. Association of PD-1/PD-L axis expression with cytolytic activity, mutational load, and prognosis in melanoma and other solid tumors. Proc Natl Acad Sci USA. 2016;113:e7769-77.

71. Wongchenko MJ, McArthur GA, Dréno B, Larkin J, Ascierto PA, Sosman J, et al. Gene expression profiling in BRAF-mutated melanoma reveals patient subgroups with poor outcomes to vemurafenib that may be overcome by cobimetinib plus vemurafenib. Clin Cancer Res. 2017;23:5238-45.

72. Hodi FS, Wolchok JD, Schadendorf D, Larkin J, Qian M, Saci A, et al. Genomic analyses and immunotherapy in advanced melanoma [abstract]. Cancer Res. 2019;79(Suppl):CT037.

73. Gerami P, Cook RW, Wilkinson J, Russell MC, Dhillon N, Amaria $\mathrm{RN}$, et al. Development of a prognostic genetic signature to predict the metastatic risk associated with cutaneous melanoma. Clin Cancer Res. 2015;21:175-83. https://doi.org/10.1158/1078-0432. CCR-13-3316.

74. Gerami P, Cook RW, Russell MC, Wilkinson J, Amaria RN, Gonzalez R, et al. Gene expression profiling for molecular staging of cutaneous melanoma in patients undergoing sentinel lymph node biopsy. J Am Acad Dermatol. 2015;72(5):780-5.e3. https://doi. org/10.1016/j.jaad.2015.01.009.

75. Morton DL, Thompson JF, Cochran AJ, Mozzillo N, Nieweg $\mathrm{OE}$, Roses DF, et al. Final trial report of sentinel-node biopsy versus nodal observation in melanoma. N Engl J Med. 2014;370:599-609.

76. Zager JS, Gastman BR, Leachman S, Gonzalez RC, Fleming MD, Ferris LK, et al. Performance of a prognostic 31-gene expression profile in an independent cohort of 523 cutaneous melanoma patients. BMC Cancer. 2018;18(1):130. https://doi.org/10.1186/ s12885-018-4016-3.

77. Spranger S, Bao R, Gajewski TF. Melanoma-intrinsic betacatenin signalling prevents anti-tumour immunity. Nature. 2015;523:231-5.

78. Peng W, Chen JQ, Liu C, Malu S, Creasy C, Tetzlaff MT, et al. Loss of PTEN promotes resistance to T cell-mediated immunotherapy. Cancer Discov. 2016;6:202-16.

79. Shin DS, Zaretsky JM, Escuin-Ordinas H, Garcia-Diaz A, HuLieskovan S, Kalbasi A, et al. Primary resistance to PD-1 blockade mediated by JAK1/2 mutations. Cancer Discov. 2017;2:188-201.

80. Sade-Feldman M, Jiao YJ, Chen JH, Rooney MS, Barzily-Rokni M, Eliane JP, et al. Resistance to checkpoint blockade therapy through inactivation of antigen presentation. Nat Commun. 2017;8:1136.

81. Busse A, Keilholz U. Role of TGF- $\beta$ in melanoma. Curr Pharm Biotechnol. 2011;12(12):2165-75. https://doi.org/10.2174/13892 0111798808437.

82. Spillane AJ, Menzies AM, van Akkooi ACJ. The landmark series: neoadjuvant systemic therapy (NAST) for stage 3 melanoma patients: a potential paradigm shift in management. Ann Surg Oncol. 2020;27:2188-200. https://doi.org/10.1245/ s10434-020-08566-8. 